\title{
EHA-ESMO Guidelines for the Diagnosis, Treatment, and Follow-up of Multiple Myeloma
}

\section{Theo Wolf}

Editorial Assistant

Citation: EMJ Hematol. 2021;9[1]:28-31.


NDAY 6 of the European Hematology Association (EHA) Virtual Congress 2021, Pieter Sonneveld, Professor of Hematology, Erasmus MC Cancer Institute, Rotterdam, the Netherlands, chaired the European Society for Medical Oncology and EHA (ESMOEHA) joint session on clinical practice guidelines for the diagnosis, treatment, and follow-up of multiple myeloma.

\section{NEWLY DIAGNOSED MULTIPLE MYELOMA}

Maria Gavriatopoulou, Assistant Professor of Therapeutics and Clinical Trials Methodology, National and Kapodistrian University of Athens, Greece, started by presenting the case of a 78-year-old male patient with a history of arterial hypertension, coronary artery disease, benign prostatic hyperplasia, and chronic obstructive pulmonary disease. The patient was diagnosed with mild anaemia by his general practitioner three months prior to being admitted to the hospital emergency department due to fatigue, bone pain, and mild fever. On admission, Gavriatopoulou noted that the patient's haemoglobin level was $8.2 \mathrm{~g} / \mathrm{dL}$; platelet and white blood cell levels were normal; serum creatinine and calcium levels were increased; albumin level was decreased; and the total protein level was within normal range. A bone marrow aspirate and biopsy were performed, showing a monoclonal plasma cell infiltration of $90 \%$ and a plasmablast infiltration of approximately 10\%. According to Gavriatopoulou, the serum M-spike was $4 \mathrm{~g} / \mathrm{dL}$, serum immunofixation was positive for IgGK, and urine-peak was $862 \mathrm{mg}$ per 24 hours. A whole-body CT found multiple Iytic lesions throughout the spine, compression fractures of the eleventh thoracic and second and fourth lumbar vertebra, and a paraosseous mass in the pelvis. Next, Gavriatopoulou highlighted the results of the fluorescence in situ hybridisation (FISH) studies: the $\mathrm{t}(4 ; 14)$ translocation was expressed in $96 \%$ of myeloma cells and the $\beta 2-$ microglobulin level was substantially elevated (9.9 mg/L). The patient was therefore classified as International Staging System (ISS) III and Revised International Staging System (R-ISS) III. Gavriatopoulou then provided an overview of the five therapeutic options available at the time: lenalidomide plus dexamethasone $(\mathrm{Rd})$; bortezomib, melphalan, and prednisone (VMP); bortezomib, cyclophosphamide, and dexamethasone (VCd); modified lenalidomide, bortezomib, and dexamethasone (VRd-lite); 
or daratumumab plus bortezomib, melphalan, and prednisone (Dara-VMP [in the context of a clinical trial]). Gavriatopoulou revealed that the patient was enrolled in the ALCYONE study and randomised to the Dara-VMP arm. If the patient progresses on Dara-VMP, potential options in the future include re-institution of bortezomib (with or without a third agent), starting Rd, adding $\mathrm{Rd}$ to daratumumab, considering lenalidomide plus dexamethasone, or switching to an isatuximab combination.

The second case presentation documented by Gavriatopoulou concerned a 69-year-old female. The patient had a haemoglobin level of $8.6 \mathrm{~g} / \mathrm{dL}$; creatinine and calcium were both within the normal range; $\beta 2$-microglobulin was increased; albumin level was decreased; and lactate dehydrogenase levels were within the normal range. The bone marrow biopsy revealed a plasma cell infiltration of $65 \%$. Furthermore, FISH testing was positive for deletion $17 p$ (in $40 \%$ of the plasma cells) and negative for translocation $t(4 ; 14)$, translocation $t(14 ; 16)$, and add1q. Wholebody low-dose CT revealed multiple lytic lesions in the thoracic and lumbar vertebrae as well as in the pelvis; however, there were no fractures or extraosseous mass. Gavriatopoulou stated that the patient (classified as ISS Stage III and R-ISS Stage III) was initially treated with bortezomib, lenalidomide, and dexamethasone (VRd) for eight cycles and achieved very good partial remission. Due to the presence of deletion 17p, the patient received maintenance with bortezomib (every 15 days) and lenalidomide, and remained in very good partial remission for 19 months. Despite this, the patient eventually relapsed, with severe anaemia, a plasma cell infiltration of $55 \%$ in the bone marrow, and skin and liver extramedullary disease. Consequently, the patient received second-line therapy with daratumumab plus carfilzomib and dexamethasone (D-Kd) and achieved haematological partial remission, disappearance of skin plasmacytomas, and a $60 \%$ reduction in liver disease. Carfilzomib was discontinued after seven cycles because of uncontrolled hypertension. The patient progressed again, with new skin plasmacytomas and an increase in liver disease. Belantamab mafodotin was utilised in the third-line setting, and the patient achieved a haematological partial remission, partial remission in liver plasmacytomas, and disappearance of skin plasmacytomas. One of the main adverse events reported was ocular toxicity, which completely resolved when treatment was interrupted and supportive care was administered. This did not re-occur upon treatment re-initiation at a reduced dose level.

\section{FRONT-LINE TREATMENT}

Francesca Gay, Associate Professor of Hematology, University of Turin, Italy, started by summarising the treatment algorithm for people with newly diagnosed multiple myeloma. The major discriminant at the time of diagnosis is eligibility for autologous stem cell transplantation (ASCT). In terms of induction therapy, transplant-eligible patients are administered either VRd or daratumumab plus bortezomib, thalidomide, and dexamethasone (Dara-VTd). If neither of these options are available, VTd or VCd can be used instead. Thereafter, the standard treatment is autologous transplant with high-dose (200 $\mathrm{mg} / \mathrm{m}^{2}$ ) melphalan. Lastly, lenalidomide is given as maintenance. In patients who are not eligible for ASCT, such as those featured in the previous presentation by Gavriatopoulou, there are three strategies considered as first-line options: Dara-Rd, Dara-VMP, and VRd. When Dara-Rd and Dara-VMP are not available, VRd is the preferred option in fit patients. Alternatively, Rd and VMP may be considered for individuals who cannot receive the previous regimens.

According to Gay, ASCT eligibility is based on a patient's age ( $>70$ years) and the absence of co-morbidities. VRd is likely to offer the best risk-benefit profile among triplets based on bortezomib; however, this treatment regimen lacks a Phase III randomised head-to-head comparison with VTd and is not licensed by the European Medicines Agency (EMA). 
lenalidomide-naïve or -sensitive patients. For example, the combination of carfilzomib, lenalidomide, and dexamethasone is associated with an improved PFS and overall survival. Moreover, Dara-Rd is an effective regimen in the second-line setting. Finally, elotuzumab- and ixazomib-Rd have been used in certain patient subpopulations (i.e., elderly individuals). However, Dimopoulos emphasised that one of the major concerns is refractoriness to lenalidomide. Data from the ENDEAVOR trial illustrated that carfilzomib in combination with dexamethasone was associated with a median PFS of approximately 9 months in patients who were lenalidomide-refractory. A similar length of PFS was observed in the CASTOR trial, where daratumumab was combined with bortezomib and dexamethasone. Clearly, these are not very promising data. Dimopoulos focused on more recent studies that have provided better results. For instance, in the OPTIMISMM trial, pomalidomide, bortezomib, and dexamethasone ( $P V d$ ) showed superior efficacy relative to bortezomib plus dexamethasone in patients with relapsed or refractory multiple myeloma previously exposed to lenalidomide. When using PVd, the median PFS for individuals progressing on lenalidomide was in excess of 18 months. According to Dimopoulos, the most robust data for this specific subset of patients have come from the CANDOR and IKEMA studies, which both have a common design and investigational arm (using carfilzomib at the standard dose of $56 \mathrm{mg} / \mathrm{m}^{2}$ with dexamethasone). In the counter-trial, there was addition of daratumumab (CANDOR) or isatuximab (IKEMA). The counter-trial showed that the median PFS for patients who progressed on lenalidomide was 28 months.

Dimopoulos also outlined possible treatment options for anti-CD38 and lenalidomide pre-treated or refractory individuals. Data from the BOSTON trial, where selinexor, bortezomib, and dexamethasone has been used, showed high response rates and low rates of peripheral neuropathy. Likewise, a combination of venetoclax, bortezomib, and dexamethasone exhibited promising clinical efficacy with acceptable tolerability and safety in the BELLINI trial.

Dimopoulos outlined the EHA-EMSO 2021 recommendations for second and subsequent relapses. Combinations of pomalidomide, lowdose dexamethasone and either isatuximab (ICARIA-MM study) or daratumumab (APOLLO study) significantly improved PFS in people with relapsed or refractory multiply myeloma. With regard to newer regimens, Dimopoulos revealed that belantamab mafodotin has been approved in the European Union (EU) for use as monotherapy in individuals who have failed all other treatment options (penta-refractory patients).

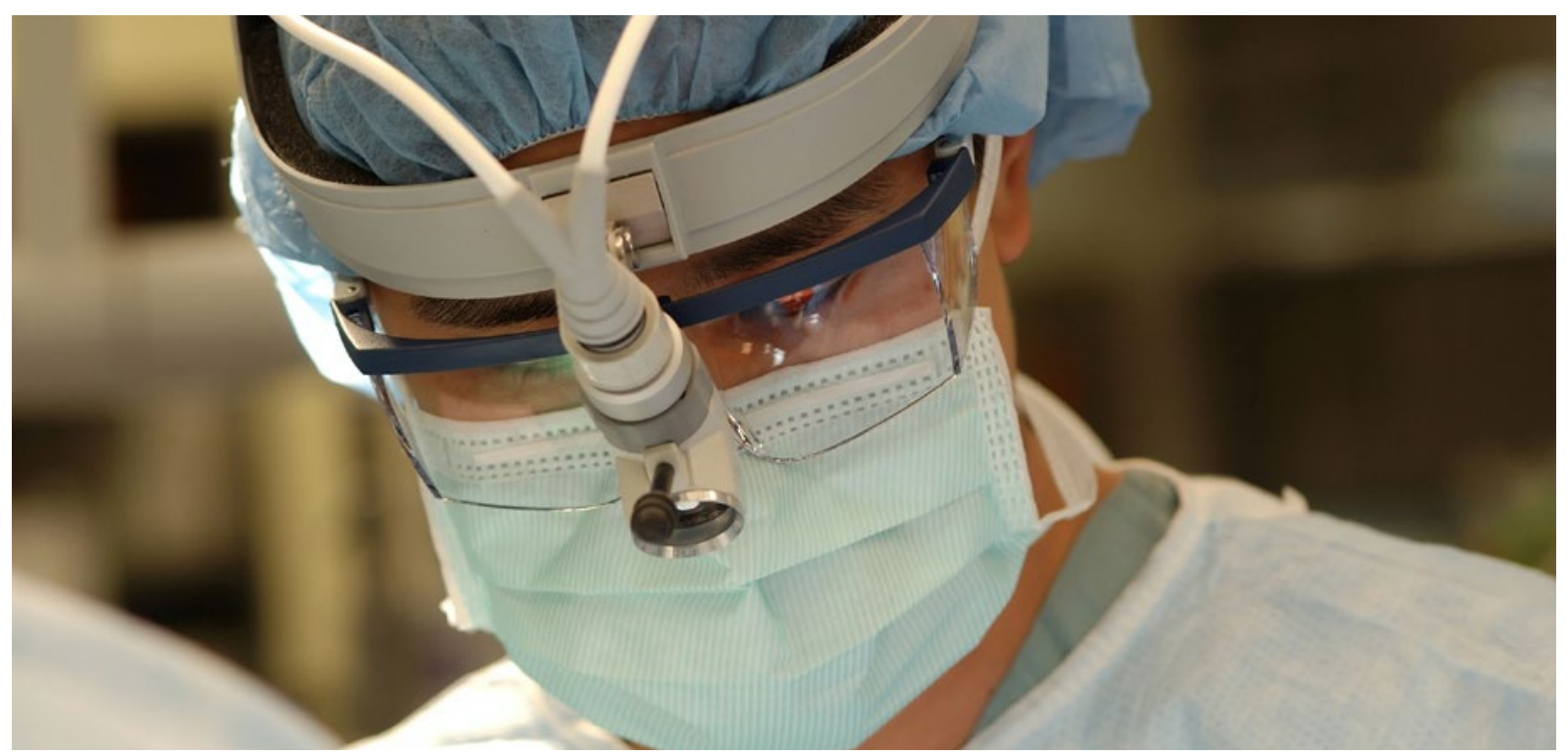

